\title{
Improvement in the Selectivity of the Chemigram Approach in Gas Chromatography/Infrared Spectroscopy
}

\author{
Dongjin Pyo* and Ken-ichi HaRADA** \\ *Department of Chemistry, Kangweon National University, Chuncheon, South Korea 200-701 \\ **Faculty of Pharmacy, Meijo University, Tempaku, Nagoya 468, Japan
}

\begin{abstract}
As gas chromatography/infrared spectrometry (GC/IR) becomes routinely available, methods must be developed to contend with the large amount of data produced. We demonstrate computer methods that can be used to determine the proper ranges of frequencies within which characteristic patterns of absorbances for a given functional group can be found. The methods can be applied to Chemigram calculations for selecting aromatic compounds from collected GC/IR data. The computer acts as a functional-group selective detector.
\end{abstract}

Keywords Chemigram, selectivity, gas chromatography/infrared spectrometry, aromatic compound

The direct coupling of a gas chromatograph to an infrared spectrometer is an important and powerful analytical tool for the analysis of complex mixtures. Since a large amount of infrared data can be produced during a gas chromatography run, a powerful computing system is desirable for providing the experimenter with capabilities of speed and automation for assistance in the analysis of the spectral data. It seems obvious that the manual methods used for compound identification, which often involve a direct interpretation of various functional groups, or a spectral comparison (standard versus unknown), are very inefficient when performed by humans. Exceptions are when the unknown is restricted to a welldefined class of materials and to cases in which the operator has had experience with the chemicals involved. Automated computer searching or interpretation schemes have therefore been needed, and various systems have been developed, such as: library searching ${ }^{1,2}$, Program for the Analysis of Infrared Spectra (PAIRS) $^{3,4}$, pattern recognition ${ }^{5,6}$, and a new expert system based on principal-component analysis (PCA). ${ }^{7}$ However, one problem with automating the procedure of searching collected gas chromatography/infrared spectroscopy (GC/IR) data for an unknown spectrum with a computer is how to reduce the size of the data in order to maximize the efficiency of the searching process. If the data system is sufficiently sophisticated, it becomes tedious and time consuming during computer searching through a large set of collected data. Although a sensible approach is to somehow select some of the entire collected data for further attention, selecting them in a way that maximizes the probability of selecting compounds is more interesting concerning the analysis at hand. All of the usual identification routines (manual interpretation, library searching or automated inter- pretation) could be applied to this smaller subset. This kind of data reduction method is a process which we refer to as selective data reduction. ${ }^{8}$

Selective data reduction involves any data-processing method that somehow selects certain data as being more "important", or more "interesting", for analyst. The selection of important data can be based on a number of criteria. Peak-finding routines are just selective data reduction using the spectral intensity as a criterion. The selection criteria often used include the presence of some spectral feature of interest. As selective data reduction routines based on spectral features in gas chromatography/mass spectrometry (GC/MS), mass chromatograms $^{9}$ and isotope cluster chromatograms ${ }^{10,11}$ have been developed. In GC/IR, "Chemigrams"12 are the most widely used selective data reduction routine to indicate the presence of absorption peaks of interest. These involve plots of integrated absorbance in a defined spectral window. The Chemigram provides a convenient way to confirm tentative fraction identifications without interpretating the complete IR spectrum. Although useful, a Chemigram has only limited selectivity, because it does not distinguish patterns of absorbances, but, rather, measures the total absorbance.

In a previous study we reported on another selective data-reduction method ${ }^{13}$ based on the presence of patterns of absorbances for a given functional group in GC/IR. In this paper we describe an improvement in the selectivity of the Chemigram approach by an automatic determination of the proper ranges of the frequencies within which the characteristic patterns of the absorbances for a given functional group will be found. To test our approach, the methods were applied to an aromatic functional group. A greater degree of selectivity was observed than in the case of simple 
Chemigram-type routines using one region.

Procedure that try to detect the presence of specific functional groups, either directly from an interferogram or after computing a Fourier transform of the absorbance spectra of the unknowns, has been developed; the latter method shows considerable promise. Chemigrams are used in one of these approaches, and can be created by monitoring all of the collected GC/IR data while monitoring the absorbances over specified infrared spectral regions as a function of time. Chemigrams have been most successful in identifying functionalities that contain characteristic peaks that are well isolated from other classes of compounds. However, one difficulty involves the determination of specified IR spectral regions, which is done more or less, manually. Our work focused on the development of an automated determination of the characteristic IR spectral regions for a certain functional group.

It is usually impossible to determine the structure of any compound in the infrared spectra on the basis of an absorption band in one spectral region. To determine the structural units present in a compound, it is therefore necessary to use group frequencies by searching the entire infrared spectrum (usually $4000-450 \mathrm{~cm}^{-1}$ ). Finding the group frequencies for each functional group is the most important step for any structural elucidation using the infrared spectra. Obviously, the more accurate are the group frequencies, the better is the structural elucidation for any compound. Once the group frequencies have been determined, the band intensities and widths for those group frequencies become very useful parameters. We have therefore developed an algorithm which can be used to calculate very accurate parameters (peak positions, peak intensity, peak width) for infrared spectra while utilizing all these band parameters to find the IR spectra/structure correlation. By using accurate band parameters, the group frequencies or characteristic regions for various functional groups can be determined.

Most Chemigram-type approaches ${ }^{12,13}$ deal with relatively simple compound classes, such as alcohols and carboxylic acids, using only one spectral region for each functional group. For example, for the selective data reduction of alcohols ${ }^{13}$, only one region $(3800-3400$ $\mathrm{cm}^{-1}$ ) and one pattern of absorbances have been used in that region. However, in the infrared spectra of compounds with more complex functional groups, it is frequently impossible to obtain structure information concerning the compound based on absorption in one region. Therefore, we introduce in this paper a new method which can deal with compounds with multiple characteristic patterns in multiple regions.

\section{Experimental}

The computer programs described were written in FORTRAN and run on an CYBER 962-31 computer (they are available from the authors). An IR database was used to provide a large number of spectra from a variety of compound types (the EPA Vapor Phase Collection of 3300 spectra, available from Dr. J. de Haseth at the University of Georgia). The spectra were stored in digital format on magnetic tape. A general information "header" record, which included the compound name, formula, molecular weight, Chemical Abstracts Service (CAS) registry number, melting point, boiling point, and Wiswesser line notation (WLN), was followed by the digital spectra, measured at $2 \mathrm{~cm}^{-1}$ resolution from 4000 to $450 \mathrm{~cm}^{-1}$. The format of the records has appeared in the literature. ${ }^{14}$

The basic strategy for carrying out Chemigram experiments involved using spectra from the database to identify specified IR spectral regions that characterize a certain functional group; those regions in a series of "unknown" spectra were then searched. Representatives of a functional group were identified by computer searching the WLN in the database header records. The list of spectra retrieved by WLN was checked against the compound names in order to avoid any coding errors. To determine proper portions of the full IR range for the given functional group, spectra in the training set were changed from their usual format of (absorbance and wavenumber) pairs to a peak parameter table. We developed computer techniques to calculate the center of a peak, the peak absorbance and the full width at half height (FWHH) of all bands in the spectrum, including those bands that are partially or completely unresolved from a neighboring spectral feature. The techique that is being used first narrows each band by Fourier selfdeconvolution (FSD), and then fitting the deconvolved spectrum (in which all significant bands have been at least partially resolved) by a series of Lorentzian bands.

\section{Results and Discussion}

We selected aromatic compounds as a compound class to test our approach. Obviously, it is necessary to examine more than one region to identify the structure of aromatic ring-containing compounds. Finding the proper characteristic regions for aromatic compounds through examining the entire infrared spectrum (4000$450 \mathrm{~cm}^{-1}$ ) is the most important step for the selective reduction of aromatic compounds. Obviously, the more characteristic is the regions, the better is the selectivity for a class of compounds. To determine the proper characteristic regions for aromatic compounds, we have employed an algorithm which can generate very accurate band parameters (peak position, peak identity, and peak width) for a given infrared spectrum. First of all, the band parameters for the vapor-phase spectra of thirty aromatic compounds are obtained. As an example, the spectrum (Fig. 1a) of isobutylbenzene (EPA database serial number 537) was converted to a peak table spectrum (Table 1) by combining Fourier selfdeconvolution and curve-fitting. Figure 1b is a synthesized spectrum comprising 23 Lorentzian peaks using the peak parameters given in Table 1. Excellent 


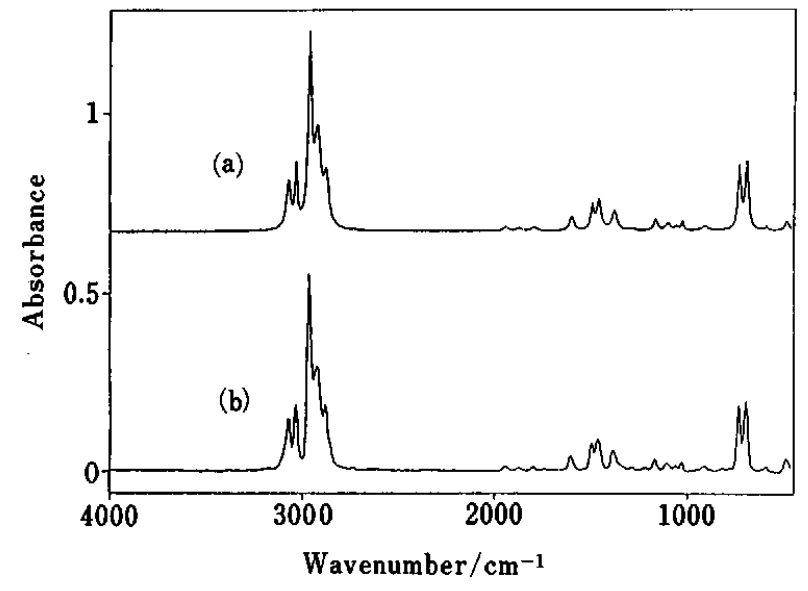

Fig. 1 IR spectra of isobutylbenzene. (a) Synthesized spectrum of 23 Lorentzian peaks from Table 1. (b) Observed spectrum of isobutylbenzene.

Table 1 Peak table spectrum of isobutylbenzene

\begin{tabular}{rcc}
\hline Center & Height (Abs.) & Width (FWHH) \\
\hline 3076.40 & 0.1367 & 27.00 \\
3035.90 & 0.1701 & 15.43 \\
2966.50 & 0.5188 & 21.22 \\
2926.00 & 0.2617 & 42.43 \\
2879.70 & 0.1321 & 30.86 \\
2735.00 & 0.0029 & 13.50 \\
1944.20 & 0.0128 & 30.86 \\
1874.80 & 0.0088 & 28.93 \\
1797.60 & 0.0119 & 30.86 \\
1602.80 & 0.0434 & 25.07 \\
1496.70 & 0.0691 & 17.36 \\
1462.00 & 0.0892 & 28.93 \\
1381.00 & 0.0581 & 30.86 \\
1288.40 & 0.0049 & 38.58 \\
1166.90 & 0.0342 & 23.15 \\
1103.20 & 0.0216 & 30.86 \\
1058.90 & 0.0113 & 17.36 \\
1029.90 & 0.0262 & 15.43 \\
921.30 & 0.0146 & 36.65 \\
734.90 & 0.1811 & 19.29 \\
696.30 & 0.1953 & 21.22 \\
592.10 & 0.0130 & 11.57 \\
486.10 & 0.0293 & 25.07 \\
\hline
\end{tabular}

matches between these two spectra were found. The band parameters for the vapor-phase spectra of the thirty aromatic compounds listed in Table 2 were obtained in the same manner.

A computer routine was written in order to search for characteristic spectral features which are common to an aromatic compound class. In this routine the peak table spectra are reformatted as binary points along the wavenumber axis, with all points one half width at half height on either side of the band center being represented as ones. All other points are represented as zeros (For a more specific spectral representation, a region for each
Table 2 List of 30 aromatic compounds as a training set (EPA Library)

\begin{tabular}{ll}
\hline 0011 & benzene, ethoxy-, \\
0026 & benzene, methyl-, \\
0033 & toluene, 3,4-dichloro-, \\
0034 & toluene, 2,4-dichloro-, \\
0035 & toluene, $p$-chloro-, \\
0040 & benzene, $t$-butyl-, \\
0075 & benzene, 1,2,4-trimethyl-, \\
0090 & benzene, $m$-diethyl-, \\
0102 & phenol, $o$-ethoxy-, \\
0146 & benzene, 1-chloroethyl-, \\
0187 & dodecane, 1-phenyl-, \\
0200 & phenol, $o$-allyl-, \\
0258 & phenol, $m$-butyl-, \\
0394 & aniline, $m$-fluoro-, \\
0305 & aniline, $p$-fluoro-, \\
0311 & benzene, 4-bromo-1-ethyl-, \\
0366 & phenol, $m$-bromo-, \\
0537 & benzene, isopropyl-, \\
0538 & benzene, ethyl-, \\
0539 & benzene, $s$-butyl-, \\
0572 & benzene, $o$-dimethoxy-, \\
0644 & phenol, $o$-isopropyl-, \\
0665 & benzene, 1,4-difluoro-, \\
0676 & benzene, 1-bromo-2-fluoro-, \\
0677 & benzylamine, $p$-isopropyl-, \\
0689 & benzyl alcohol, $m$-methoxy-, \\
0799 & benzene, 1,3,5-trimethyl-, \\
0902 & benzene, butyl-, \\
0981 & benzene, isobutyl-, \\
2525 & benzonitrile, 2,4-dimethyl-, \\
\hline
\end{tabular}

band that is bounded by a fraction of the HWHH can obviously be selected.). As an example, the highwavenumber region of the spectrum of isobutylbenzene is shown below:

$$
\begin{aligned}
& 000 \ldots .001111 \ldots \ldots .111100000 \ldots .000001111 \ldots .111110000 \text { etc. }
\end{aligned}
$$

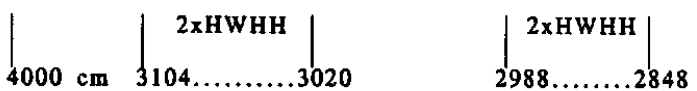

$$
\begin{aligned}
& \text { (start) (Aromatic C-H Stretch) (Alkane C-H Stretch) }
\end{aligned}
$$

By overlapping the spectra that have been formatted in this way, characteristic regions for a given functional group can be selected by finding the regions common to that class of spectra, i.e., the regions where each spectrum contains ones. An occurrence value for every wavenumber can be calculated simply by adding the binary spectra, so that if an occurrence value equal to the number of spectra is computed in a given region, that region would be a candidate for the spectra/structure correlation table. Using the binary spectral data for thirty aromatic compounds whose reference spectra were available in the EPA library of vapor-phase infrared spectra, the occurrence values for every wavenumber were calculated by overlapping and summing these thirty binary spectra. 
Table 3 Characteristic region search results for aromatic compounds with thresholds of $100 \%$ and $93 \%$

\begin{tabular}{cccc}
\hline & $100 \%$ & & $93 \%$ \\
\hline 1. & $3057-3051 \mathrm{~cm}^{-1}$ & 1. & $3089-3034 \mathrm{~cm}^{-1}$ \\
2. & $1610-1598 \mathrm{~cm}^{-1}$ & 2. & $1620-1581 \mathrm{~cm}^{-1}$ \\
3. & $1500-1494 \mathrm{~cm}^{-1}$ & 3. & $1504-1479 \mathrm{~cm}^{-1}$ \\
& & 4. & $1064-1062 \mathrm{~cm}^{-1}$ \\
& & 5. & $1053-1051 \mathrm{~cm}^{-1}$ \\
\hline
\end{tabular}

Results (Table 3) were obtained with threshold occurrence values of $100 \%$ (i.e., all of the aromatic compounds absorbed in this region) and $93 \%$ (i.e., 28 of the 30 aromatic compounds absorbed in this region). As would be expected, broader regions were calculated with a threshold occurrence value of $93 \%$ compared with a threshold occurrence value of $100 \%$; additional spectral regions $\left(1068-1058 \mathrm{~cm}^{-1}\right.$ and $\left.1056-1051 \mathrm{~cm}^{-1}\right)$, corresponding to the in-plane bending vibrational modes of the $\mathrm{C}-\mathrm{H}$ moiety, were added.

Once the characteristic regions of aromatic compounds are determined, Chemigram calculations can be made by summing the absorbances over those regions. Those regions must be general enough that all members of the compound class have absorption peaks, but selective enough that most of the other functional groups do not have absorption peaks, since the goal of the Chemigram is simply to screen a large number of spectra in a short time, and to determine for each spectrum whether it "probably does" or "probably does not" contain absorption peaks of interest.

First, as a control, we integrated the absorbance in the $3100-3000 \mathrm{~cm}^{-1}$ window for the first 200 spectra in the database. This comprised information that we could usually obtain in a simple Chemigram experiment for the aromatic functional group. Figure 2a shows the percentage of compounds found (either aromatics or nonaromatics) vs. $\Sigma S_{\mathrm{j}}$. By choosing a threshold value of 3.20 , the vast majority of the aromatics could be retrieved, while only a small fraction of the nonaromatics was included. If the maximum selectivity is sought, one would select a threshold at which the differences between the two curves in Fig. 2a is greatest. In this case, when the threshold value is 3.20 , the greatest difference is shown. At this threshold value, only $72 \%$ of the aromatic compounds were retrieved; $19 \%$ of the non-aromatic compounds were also retrieved (Fig. 2a). The spectra of the non-aromatic compounds that were assigned $\Sigma S_{\mathrm{i}}>3.20$ were examined in order to find out why they had been found. Not surprisingly, in most cases, there were alkenes such as 2-methyl-2-butene (database serial number 41), and 2,4,4-trimethyl-2pentane (database serial number 18), allyl chloride (database serial number 16). These results seem to be understandable since it has been well known ${ }^{15}$ that alkanes and aromatics involve $\mathrm{C}-\mathrm{H}$ stretching at frequencies higher than $3000 \mathrm{~cm}^{-1}$, whereas alkane groups

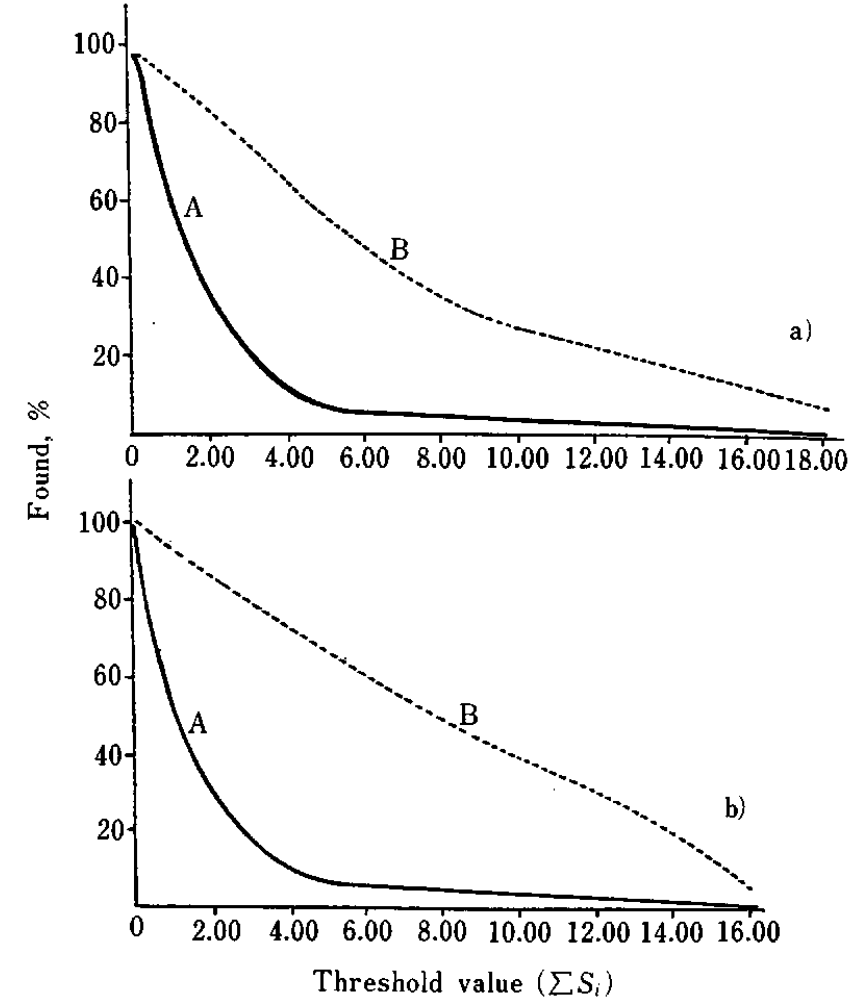

Fig. 2 Chemigram search results of aromatic compounds: a) search region, $3100-3000 \mathrm{~cm}^{-1}$; b) search regions, 3089 $3024 \mathrm{~cm}^{-1} ; 1608-1587 \mathrm{~cm}^{-1} ; 1500-1483 \mathrm{~cm}^{-1}$. Percentage A: percentage of non-aromatic compounds (-) with a $\Sigma S_{\mathrm{i}}$ value greater than the threshold shown; percentage $B$ : percentage of aromatic compounds $(\cdots)$ with a $\Sigma S_{\mathrm{i}}$ value greater than the threshold shown.

involve frequencies lower than $3000 \mathrm{~cm}^{-1}$. Other cases were chlorine-containing saturated alkanes, such as 1,4dichlorobutane (database serial number 29) and 2chlorobutane (database serial number 117). These results also show that $\mathrm{C}-\mathrm{H}$ stretching absorption in $-\mathrm{CH}_{2}$ - halogen compounds is shifted by about $50 \mathrm{~cm}^{-1}$ to a higher wavenumber, compared to saturated alkane compounds. ${ }^{15}$

Similarly, the spectra of the aromatic compounds that were not assigned $\Sigma S_{\mathrm{i}}>3.20$ were examined. In many cases, the problem was a low intensity of bands in the aromatic $\mathrm{C}-\mathrm{H}$ stretching region. These include isobutyl ester of benzoic acid, ethyl ester of $p$-toluic acid, and 3-bromoacetophenone. This phenomenon has shown ${ }^{16}$ that the intensities of the $\mathrm{C}-\mathrm{H}$ stretching band adjacent to the $\mathrm{C}=\mathrm{O}$ groups in ketones and esters involves a decrease, while the deformation $\mathrm{C}-\mathrm{H}$ band intensities show an increase. Other cases, such as $\alpha, \alpha, \alpha, 4-$ tetrachlorotoluene (database serial number 31 ) and $\alpha, 2,4$-trichlorotoluene (database serial number 64), showed low $\Sigma S_{\mathrm{i}}$ values of 0.03 and 1.48, each. It is also known that electron-attracting substituents on the aromatic ring decrease the $\mathrm{C}-\mathrm{H}$ stretching intensities; ${ }^{17}$ these results were obtained by monitoring the absorbance over only one spectral region $\left(3100-3000 \mathrm{~cm}^{-1}\right)$. 
These characteristic regions $\left(3089-3024 \mathrm{~cm}^{-1}, 1608\right.$ $1587 \mathrm{~cm}^{-1}$, and $1500-1483 \mathrm{~cm}^{-1}$ ) for aromatic functional groups generated from our characteristic region generating routines were used to calculate Chemigrams. We integrated the absorbances in each of the three regions, and then summed the total absorbances. The results are given in Fig. 2b. Two curves show the percentages of aromatic compounds and non-aromatic compounds found at a certain threshold value $\left(\Sigma S_{\mathrm{i}}\right)$. As expected, better results, i.e. greater difference between two curves, were obtained. By choosing a threshold value of 3.40 , $78 \%$ of the aromatic compounds were retrieved, while only $16 \%$ of the non-aromatic compounds were included. Although this threshold value varies with the functional group and number of characteristic regions, a graph (such as Fig. 2) is easy to construct, and the proper value of the threshold can be determined from it. If it is important to miss none or very few of the target compounds, it is possible to choose a high threshold and to tolerate an increased occurence of false positives.

Because $\mathrm{C}=\mathrm{C}$ stretching vibration regions (1608 $1587 \mathrm{~cm}^{-1}, 1500-1483 \mathrm{~cm}^{-1}$ ) were also examined as well as $\mathrm{C}-\mathrm{H}$ stretching region $\left(3089-3024 \mathrm{~cm}^{-1}\right)$, halogencontaining aromatic compounds, such as $\alpha, \alpha, \alpha, 4$-tetrachlorotoluene (database serial number 31) and $\alpha, 2,4-$ trichlorotoluene (database serial number 64), which have a low intensity of $\mathrm{C}-\mathrm{H}$ stretching bands, showed high $\Sigma S_{\mathrm{i}}$ values (i.e., 5.14, 8.55 each). In Fig. 2b, the maximum discrimination is found at a threshold of $\Sigma S_{\mathrm{i}}=3.40$. Those halogen-containing aromatic compounds were thus assigned as aromatics, since their $\Sigma S_{\mathrm{i}}$ values are greater than 3.40 .

One aspect of the Chemigram approach deserves comment: in a Chemigram, since one only calculates the integrated absorbances over the given spectral windows the pattern of absorbances does not matter. Therefore, a Chemigram has limited selectivity. In our laboratories, in order to improve the selectivity of a Chemigram, methods used to compare the pattern of absorbance in an unknown spectra with the average pattern of aromatic compounds are being developed.
In conclusion, a proper determination of the characteristic regions for a functional group can improve the selectivity of a Chemigram. Such a Chemigram can be used to select spectra which would warrant further interpretation, whether that interpretation be by use of library searching, artificial intelligence, or manual spectral identification.

\section{References}

1. G. T. Rasmussen and T. L. Isenhour, Appl. Spectrosc., 33, 371 (1979).

2. M. D. Erickson, Appl. Spectrosc., 35, 181 (1981).

3. M. A. Puskar, S. P. Levine and S. R. Lowry, Anal. Chem., 58, 1156 (1986).

4. L. Ying, S. P. Levine, S. A. Tomellini and S. R. Lowry, Anal. Chem., 59, 2197 (1987).

5. K. Varmuza, Anal. Chim. Acta, 122, 227 (1980).

6. M. P. Derde and D. L. Massart, Anal. Chim. Acta, 191, 1 (1986).

7. J. H. Perkins, E. J. Hasenoehrl and P. R. Griffiths, Anal. Chem., 63, 1738 (1991).

8. R. J. Anderegg, Am. Lab. (Fairfield, Conn.), 17, 20 (1985).

9. R. A. Hites and K. Biemann, Anal. Chem., 42, 855 (1970).

10. J. L. Laborosse and R. J. Anderegg, J. Chromatogr., 314, 83 (1984).

11. R. J. Anderegg, Anal. Chim. Acta, 176, 175 (1985).

12. D. R. Mattson and R. L. Jukian, J. Chromatogr. Sci., 17, 416 (1979).

13. R. J. Anderegg, D. Pyo, Anal. Chem., 59, 1914 (1987).

14. P. R. Griffiths, L. V. Azarraga, J. A. de Haseth, R. W. Hannah, R. J. Jakobsen and M. M. Ennis, Appl. Spectrosc., 33, 543 (1979).

15. K. Nakanishi, "Infrared Absorption Spectroscopy", p. 20, Holden-Day Inc., San Francisco, 1962.

16. S. A. Francis, J. Chem. Phys., 19, 942 (1951).

17. M. St. C. Flett, J. Phys. Radium., 15, 388 (1954).

(Received July 11, 1992)

(Accepted November 11, 1992) 